\title{
Laser ranging measurements of turbulent water surfaces
}

\author{
Gašper Rak ${ }^{1 *}$, Franc Steinman ${ }^{1}$, Marko Hočevar ${ }^{2}$, Matevž Dular ${ }^{2}$, Matija Jezeršek $^{2}$ and Urban Pavlovčič

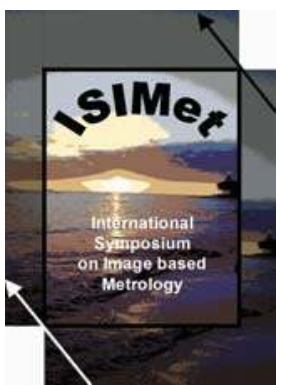 \\ ISIMet 2 \\ International \\ Symposium on \\ Image based \\ Metrology \\ Hawaii, Maui \\ December 16-21 \\ 2017 \\ Abstract \\ Laser ranging is a measurement method, applied in a wide range of applications. In this \\ study, laser ranging is used to measure the height of turbulent water flows. Measurements \\ were performed in three cross sections of a confluence where a tributary flow meets the \\ main flow. Both flows exhibited high Reynolds and Froude numbers where the free-water \\ surface profiles were turbulent, non-stationary and non-homogeneous. Measurements were \\ taken using a commercial LIDAR and a high-speed camera. The high-speed camera was \\ operated on the principle of laser triangulation, using only the illumination from the LIDAR \\ laser beam. Since, no other state-of-the-art method for measuring instantaneous water \\ surface profiles exists, LIDAR and triangulation methods could only be compared with each \\ other. The results show good agreement between both methods for the average turbulent \\ water profile and fair agreement for instantaneous profiles. Presented herein is an \\ explanation of these results.

\section{Keywords} \\ LIDAR — laser triangulation — free surface — turbulence — non-stationary surface — high-speed \\ camera \\ ${ }^{1}$ University of Ljubljana, Faculty of civil and geodetic engineering, - \\ ${ }^{2}$ University of Ljubljana, Faculty of mechanical engineering, Ljubljana, Slovenia \\ *Corresponding author: gasper.rak@fgg.uni-lj.si
}

\section{Introduction}

Water surface measurements of turbulent free surface flows are an important part of hydraulic measurements. Such flows are encountered in a wide range of applications in civil, chemical, environmental, mechanical, mining and nuclear engineering. In turbulent free surface flows, air bubble entrainment is the result of surface deformation. When the turbulent shear stress is greater than the surface tension stress that resists the interfacial breakup, bubble entrainment is possible. The water /air interface has been studied experimentally, numerically and theoretically over the last few decades, and a good overview is given by $\mathrm{H}$. Chanson in [1].

Distance measurements to various objects are often conducted using laser ranging methods due to their inherent noncontact and high-speed capabilities. Laser ranging is based on interferometry, triangulation or time-offlight methods [2]. This study considers only the latter two methods. For time-of-flight measurements, LIDAR (Light Detection And Ranging) is one of the most widely used and promising remote sensing technologies [3; 4]. A LIDAR's main components include a laser, scanner and optics, and a photodetector and receiver electronics. Its application in many different fields of science and engineering, among them agriculture, archaeology, surveying, autonomous vehicles, robotics, military, atmospheric remote sensing, and meteorology reflects its robustness and versatility.

Despite this, LIDAR has rarely been used for making surface water profile measurements. Blenkinsopp et al. [5] used LIDAR to measure the time-varying free-surface profile across the swash zone. In the swash zone, the water exhibits bubbles on the surface, which increases the probability of diffuse reflections and hence the possibility to make measurements using LIDAR. Their results were in agreement with measurements made using ultrasonic sensors. In addition, both Allis and Blenkinsopp independently applied the method to make laboratory based profile measurements of time-varying free-surface propagating waves [6;7], while others have used LIDAR to characterise water surfaces using LIDAR based bathymetry. Westfeld et al. [8], for example, recently used LIDAR bathymetry to investigate the influence of ocean wave patterns on the accuracy of 3D underwater point coordinates.

Laser triangulation measurements of surfaces are common [2], but work relating to making triangulation surface water measurements of highly turbulent and aerated flows is at best limited. Mulsow et al. $[9 ; 10]$ used a modified triangulation method capable of measuring a reflected laser line. Other optical methods [11], such as particle image velocimetry and stereo vision photogrammetry or acoustic methods like Doppler velocimetry are more common but fail to provide measurements for highly aerated turbulent flows. Conventional methods remain the most commonly used for measuring water levels, including for example, resistance-type probes [12], U-manometers [13], point gauges, and ultrasonic sensors [14].

In this study, LIDAR and laser triangulation using a high-speed camera were used to measure the height of turbulent water surfaces with highly-aerated flows and high Reynolds and Froude numbers. By simultaneous using both methods, a deeper understanding of how the laser beam is scattered by the turbulent aerated open surface and how the data is interpreted can be obtained. In addition, a comparison of both measurement results is provided together with an interpretation of the measurements obtained. 


\subsection{Flow properties at the confluence}

Confluences occur in streams (natural and artificial river channels, and torrents), as well as in various types of facilities and infrastructure (fish ways and during drainage of surface water from impervious surfaces). At such a confluence, especially one with incoming supercritical flows, a distinctively three-dimensional flow of water forms and exhibits a non-stationary and non-homogeneous profile of a turbulent free-water surface. The non-stationary structure of the water flow is in both the transversal and longitudinal directions, however, studies have largely neglected to measure turbulent water surface profiles in the transversal direction in confluences composed of channels with supercritical flows $[12 ; 15 ; 16]$. The reasons for this are the limitations of conventional measurement methods (piezometers, ultrasonic sensors, point gauges, etc.), which do not allow dynamic measurements with high spatial resolution.

\subsection{Laser ranging for profiling turbulent water surfaces}

In comparison with other methods, laser ranging of turbulent water surfaces is limited due to specular reflections. In such cases, the laser beam is reflected away from the photodetector and is not measured. A diffuse surface will reflect at least some of the laser light back to the photodetector allowing the distance to the surface to be measured provided the intensity of the reflected laser light is above a certain threshold.

The use of laser ranging methods for measuring nonstationary and non-homogeneous open surface flows presents several challenges, among which are successive specular reflections, non-equidistant sampling, thresholding of the returned laser light, and problems of unwanted reflections from channel walls. In this respect, the specular reflection of a LIDAR beam from the air/water interface represents the most important factor relating to laser ranging measurements. In addition, refraction effects of the LIDAR pulse passing the air/water and water/air interfaces must be taken into account. This includes the reduced velocity of laser light in water, being approximately $75 \%$ of its velocity in air. The height of the water surface of highly turbulent open surface flows, similar to the confluence flow used in this study (Fig. 1), is difficult to determine. This is due to an abundance of droplets above the surface that can overestimate surface height, numerous steep waves that prevent measurements of concave surfaces, entrapped bubbles below the surface that can underestimate surface height, and the presence of foam that can increase measurement uncertainty. The surface is also spatially non-homogenous and non-stationary in time.

In this study, the same LIDAR beam was used for laser triangulation measurements using a high-speed camera. An assumption is made that in tap water (used in the experiment) all laser beam reflections are from the water/air interface and that they are all specular. This assumption is justified given the limited number of impurities present in tap water. Due to the turbulent nature of the surface flow, the laser beam may be reflected many times, before it reaches either the laser scanner or the high-speed camera. In the high-speed camera images, this appears as regions of bright pixels. Since individual reflections are specular and the laser light reflected to the high-speed camera cannot reach the laser scanner or receiver (and vice versa), multiple consecutive reflections were treated as a statistical process. In this way, a high number of consecutive specular reflections exhibit the same behaviour as near-diffuse reflections. A near-diffuse reflection is similar to real-diffuse reflection, although on a much larger spatial scale [17]. For real diffuse reflections most of the scattered light originates from scattering centres beneath the surface [17], and involves a series of consecutive multiple partial reflections. In this study, LIDAR and the laser triangulation high-speed camera are used to detect near-diffuse reflections, although the sensors are located at different locations.

\section{Measurements}

The location of the measuring station is shown in Figure 2. The measuring station is an open channel confluence designed for hydraulic model experiments [12; 15]. The main flow and tributary flow are at $90^{\circ}$ to each other and has sharp edges. The main channel is $6 \mathrm{~m}$ in length, while the main and tributary channels upstream of the confluence are each $1 \mathrm{~m}$ in length. The channel width is $0.5 \mathrm{~m}$ for both the main and tributary flows. All sides are rectangular and made from glass. The bottom of the measuring station is horizontal. All joints were carefully manufactured to avoid local flow separation.

The system works as follows: a centrifugal pump is used to keep a constant hydraulic head reservoir filled with tap water. From this reservoir, tap water flows by gravity to the measuring station (Fig. 2), where the flow rates were measured in both channels using ABB Watermaster electromagnetic flowmeters. The volume flow rates were set using valves while flaps, mounted at the outflow of the pressure vessels, were used to select the height of the main and tributary flows. After passing the measuring station, the water then flows into the lower reservoir. Unlike other authors [6; 7], who used laser scanning to measure timevarying free-water surface profiles of wave propagation in a wave flume, in this study no additives were used to improve reflection.

\subsection{Measuring equipment}

The measuring equipment consisted of a commercial LIDAR device, a high-speed camera and a secondary camera. The instruments were setup as shown in Figure 2. 


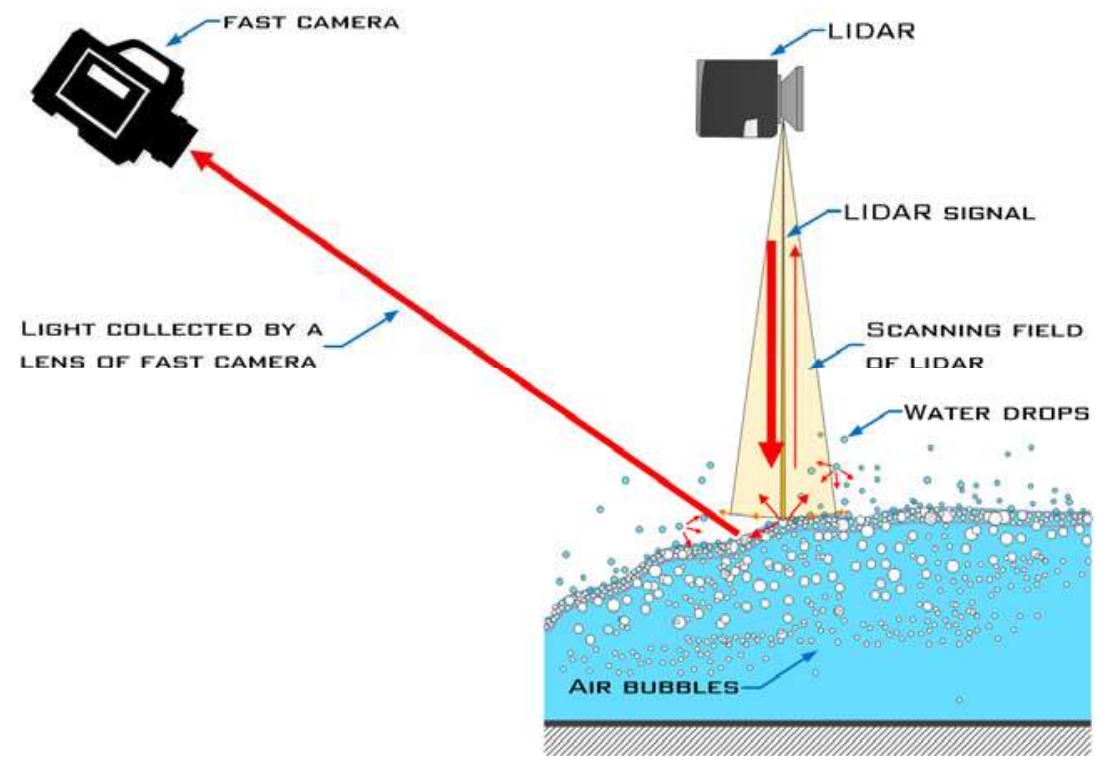

Figure 1. Specular reflections at the turbulent water surface.

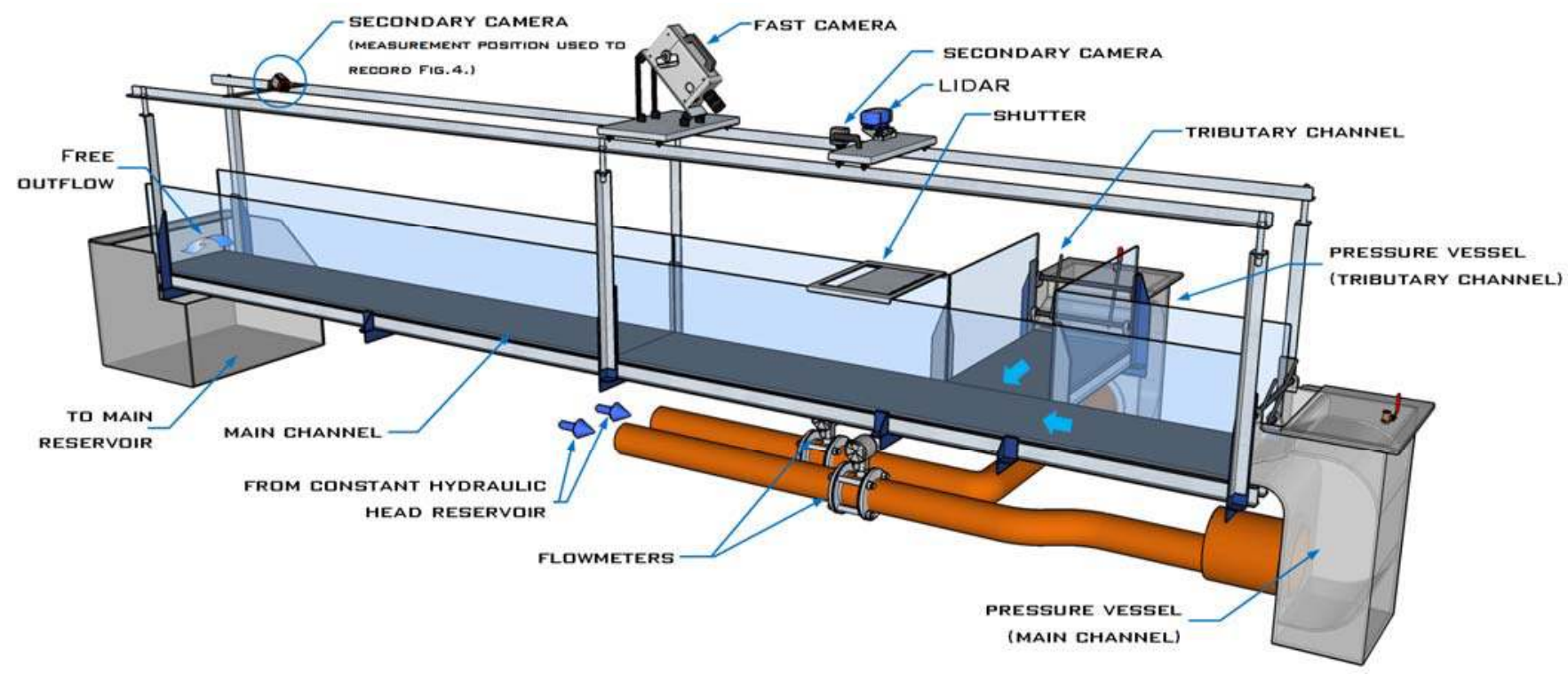

Figure 2. Measuring station

The LIDAR was mounted perpendicular above the measuring station $1 \mathrm{~m}$ above the bottom of the channel. The LIDAR was used was a Sick LMS400, chosen on the basis of an analysis made in [18]. To the authors' knowledge, no commercially available LIDAR has been designed to make water surface measurements, but among those available, the Sick LMS400 proved the most promising due to its high frequency of operation narrow laser beam and high precision [18]. The LIDAR was operated with a line scanning frequency of $270 \mathrm{~Hz}$ and an angular resolution of $0.2^{\circ}$, totalling 94500 distance measurements per second ( 270 profiles per second $x 70$ degrees field of measurement $\times 5$ measurements per degree $-0.2^{\circ}$ ). All measurements were recorded in raw format as distance/angle pairs with and without averaging and post-processing, since this would prohibit a real time comparison of LIDAR and the camera measurements. The LIDAR Sick LMS400 operates in visible red light $(\lambda=650 \mathrm{~nm})$. According to the manufacture's data, systematic measurement and statistical measurement uncertainty is $\pm 4 \mathrm{~mm}$ and $\pm 3 \mathrm{~mm}$, respectively. The beam diameter is $1 \mathrm{~mm}$.

The LIDAR data were transmitted to the measuring computer using an Ethernet connection. A dedicated driver and communication program was written using the Labview National Instruments software package, which enabled reliable LIDAR data acquisition and storage to a local disk without losing any measurement data. The LIDAR was calibrated using a strip of white paper, attached to the bottom of the channel.

The laser triangulation method was composed of a high-speed camera mounted downstream of the channel and a laser scanning projector provided by the LIDAR (Fig. 2). The camera was mounted facing upstream at a downward angle of $35^{\circ}$. The camera was a Photron SA - Zs operated 
at a framerate of 100000 frames/s at a resolution of $640 \times 280$ pixels. Each recorded high-speed camera image corresponds approximately to a single LIDAR distance measurement with a $0.2^{\circ}$ angular resolution. The lens was a F-mount $28 \mathrm{~mm} \mathrm{f} / 1.8 \mathrm{G}$ Nikkor lens fitted with a red filter (cut-off wavelength at $600 \mathrm{~nm}$ ) to filter out the blue light used by the secondary camera. The calibration procedure for the triangulation method was performed according to Jezeršek and Možina [19]. This configuration enables a direct comparison of both sets of measurements. The LIDAR laser beam also provides the illumination for the high-speed camera and each single LIDAR distance measurement corresponds to a single high-speed camera frame. Thus, an entire LIDAR scan line (scanned at $270 \mathrm{~Hz}$ ) corresponds to 350 high-speed camera images. Each recorded image is illuminated in just one position, corresponding to the position of the laser beam (Fig. 7). The time from one sample point to the other is the same for both measurements. Unlike the first and the last point of the profile (Fig. 6), all corresponding positions were recorded simultaneously using both measurement methods.

To obtain visual information about the flow, a secondary black and white camera (Fastec Hispec 4) was oriented normal to the water surface. The diffuse illumination for the secondary camera consisted of a large array of LEDs with a total power of $100 \mathrm{~W}$ at wavelength of $465 \mathrm{~nm}$. The secondary camera used a C-mount lens with a $35 \mathrm{~mm}$ focal length and recorded images at a frequency of $270 \mathrm{~Hz}$ equivalent to the scanning rate of the LIDAR. The image resolution was $1696 \times 360$ pixel and the exposure time was $3.7 \mu \mathrm{s}$. The secondary camera recorded light between $465 \mathrm{~nm}$ and $650 \mathrm{~nm}$, i.e. the blue LED and the red LIDAR laser beam.

Measurement duration for each cross section was $2 \mathrm{~s}$. During this period, the laser scanner recorded 540 line scans with a total of 189000 distance measurements, while the high-speed camera recorded 200000 images and the secondary camera 540 images. All the equipment was synchronised using a mechanical shutter mounted above the measuring station channel between the water surface and the LIDAR with secondary camera. Prior to acquisition, the shutter was closed, such that the laser beam from the LIDAR was cut and the laser triangulation high-speed camera images were dark. The LIDAR, high-speed camera and secondary camera were then started. The opening of the shutter enabled the acquisition and recording of all three sensors simultaneously. The entire experiment was performed in the dark.

\subsection{Selection of operating point and measuring positions}

A single set of operating parameters was selected for analysis (Table 1) and measurements were obtained in three measurement cross sections: CS1-CS3 (Fig. 3). Measurement cross sections were located $900 \mathrm{~mm}$ (CS1), $1100 \mathrm{~mm}$ (CS2) and $1300 \mathrm{~mm}$ (CS3) downstream from the confluence.

Reynolds number gives the ratio of inertial forces to the viscous forces within a fluid. Reynolds numbers Re were
Laser ranging measurements of turbulent water surface- 3 calculated as follows:

$\operatorname{Re}=\frac{\rho v h}{\mu}$

where $\rho$ is water density, $v$ is water velocity, $h$ is water height and $\mu$ is water viscosity. The Froude number Fr gives the ratio of the flow inertia to the external gravity field. Froude numbers were calculated using the following equation:

$\operatorname{Fr}=\frac{v}{\sqrt{g h}}$

where $g$ is gravitational acceleration.

Table 1: Operating parameters.

\begin{tabular}{|l|c|}
\hline variable & value \\
\hline main flow flow rate [l/s] & 35.5 \\
\hline tributary flow flow rate [l/s] & 26.6 \\
\hline main flow height [m] & 0.02 \\
\hline tributary flow height [m] & 0.02 \\
\hline main flow Re [-] & $7.1 \times 10^{4}$ \\
\hline tributary flow $\operatorname{Re}[-]$ & $5.3 \times 10^{4}$ \\
\hline main flow Fr [-] & 8 \\
\hline tributary flow Fr [-] & 6 \\
\hline
\end{tabular}

Figure 4 show consecutive sample images of the confluence flow at the selected operating parameters. The images reveal a highly turbulent flow.

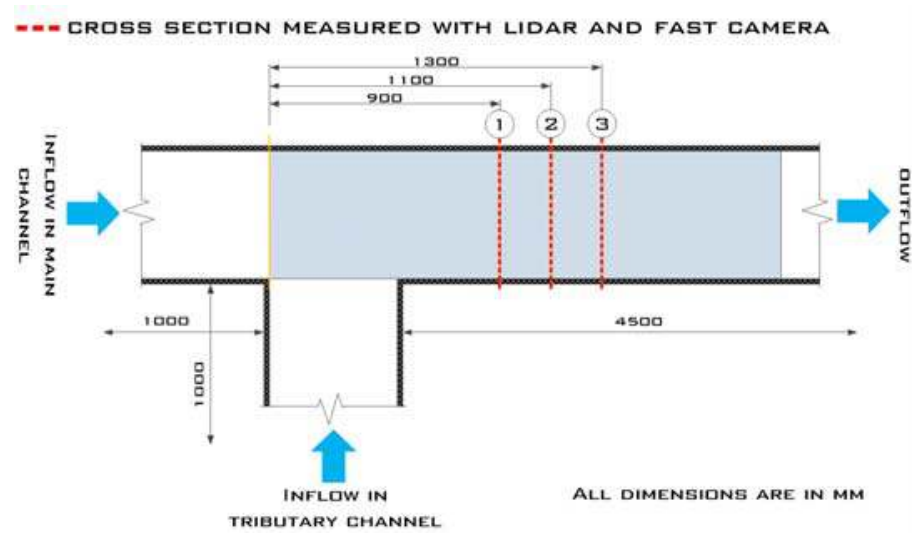

Figure 3. Dimensions of the confluence and locations of the measurement cross sections CS1, CS2 and CS3. All measurements are in $\mathrm{mm}$.

Figure 4 shows a sample image recorded using the secondary camera. In a separate experiment the secondary camera was moved to a new location indicated in Fig. 2. A framerate of $50 \mathrm{~Hz}$, a shutter speed of $500 \mu \mathrm{s}$ and additional blue LED illumination of the entire downstream section of the confluence were used. The images reveal a 
confluence flow that is highly turbulent with large amounts of air trapped. Turbulent vortices within the flow with high local velocity that are caused by steep waves and a large variation in height. Turbulent structures on the water surface are non-linear and three-dimensional and include oscillations, ridges, vortex roll-ups, wakes, hairpin like structures, turbulent bursts and flying water droplets. The turbulent structures range in size from a few $\mathrm{mm}$ to much larger structures.

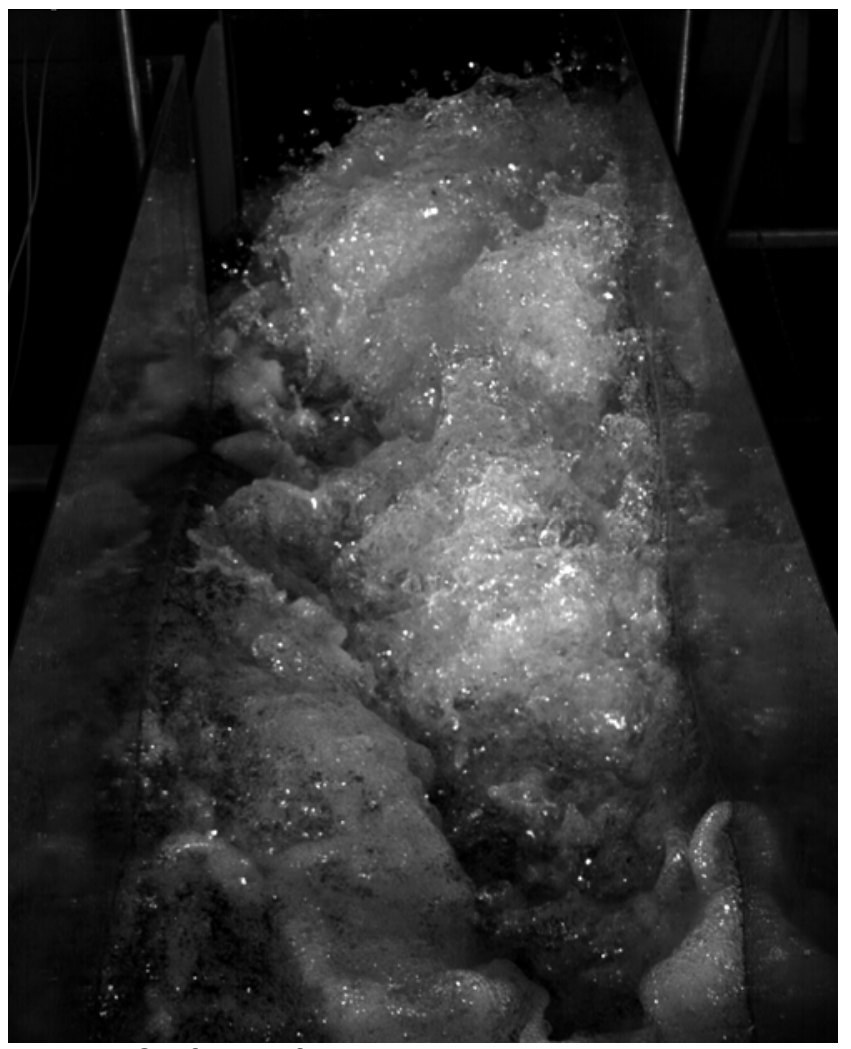

Figure 4. Confluence flow is highly turbulent, here recorded with secondary camera from position shown in Fig. 2.

\section{Data analysis}

The LIDAR data consists of a set of angle/distance pairs in the sensor's radial coordinate system. Each distance measurement was converted into two-dimensional Cartesian coordinates. To enable comparison with results of laser triangulation measurements, a linear interpolation between consecutive measurement points was performed in order to create an equidistant grid of data in a Cartesian coordinate system.

The laser triangulation high-speed camera images were analysed using algorithms, developed in LabView and C++ programming languages. The images were first filtered using a Gaussian filter. The dimensions of the filter kernel were $4.5 \%$ of the image size in the horizontal and $1.7 \%$ in the vertical direction. This allowed the intensities of specular reflections from multiple neighbouring locations to be merged together. The intensities (black and grey points in Fig. 7) represent the merging of the LIDAR beam reflections
Laser ranging measurements of turbulent water surface- 4 towards the high-speed camera and mapping to the $2 \mathrm{D}$ frame of the measurement cross sections CS1 to CS3. The location of the reflected laser light was determined as the location of maximal intensity in the image. Finally, the location of the point in the 3D space was reconstructed using triangulation [19]. It remains to be confirmed if such an approach is valid i.e., if the maximum of intensity location is sufficiently stable to reduce a $2 \mathrm{D}$ area to a single surface height value. Unfortunately, no other state-of-the-art method enabling a direct comparison of instantaneous water surface profiles exists and further research is necessary. Despite this, insight might be gained from a statistical analysis of the measurement results.

It is assumed that all measured locations correspond to the water's surface, since there are no reflections in the clear water. The only locations from where reflections could be recorded are from the flying droplets and submerged entrapped bubbles but these were neither evaluated nor taken into account. The LIDAR and laser triangulation measurements were synchronised by detecting and deleting the images and LIDAR distance measurements, where the mechanical shutter was detected.

\section{Results and discussion}

Figure 5 shows the average turbulent water surface heights (solid line) and corresponding standard deviations (dotted line, $\pm 1 \sigma$ ). The measurements results for CS2 and CS3 show remarkably good agreement between those obtained by LIDAR (blue) and laser triangulation (red). Except for the regions near the walls, both measurements agree $( \pm 10 \mathrm{~mm})$ in the channel cross section. On average, LIDAR measures a slightly greater depth (Fig. 5: blue line). Such an agreement was achieved for each location in the cross section. Compared to CS2 and CS3, the agreement between both methods is slightly worse for CS1, where the deviation is within $\pm 25 \mathrm{~mm}$. This is emphasised on the left side of the channel, up to approximately $300 \mathrm{~mm}$ out from the channel wall. The sample image from the secondary camera (Fig. 5: right image) for CS1 reveals high flow dynamics and turbulence in this region. Turbulent vortices caused by the confluence interact and form unsteady flow structures that cause surface fluctuations and air entrapment. In all of the cross sections (Fig. 5: right) such behaviour is more pronounced on the left side (up to $x=250 \mathrm{~mm}$ ).

Measurement results near the channel walls exhibit a higher measurement uncertainty in CS1 in comparison with the central region. The wall region, affected by such behaviour, extends out to approx. $50 \mathrm{~mm}$ from the walls. This localisation was more apparent for CS2 and CS3, which had less flow dynamics than CS1. Due to poor spatial localisation of the triangulation method compared with the LIDAR method, the results near both walls i.e., for $50 \mathrm{~mm}$ out for the triangulation method and $25 \mathrm{~mm}$ in the case of LIDAR, are omitted (Fig. 5). Additional reflections were also observed from the glass walls, which affected the performance of the laser triangulation high-speed camera detector and both the image analysis algorithm and the LIDAR internal algorithm. 

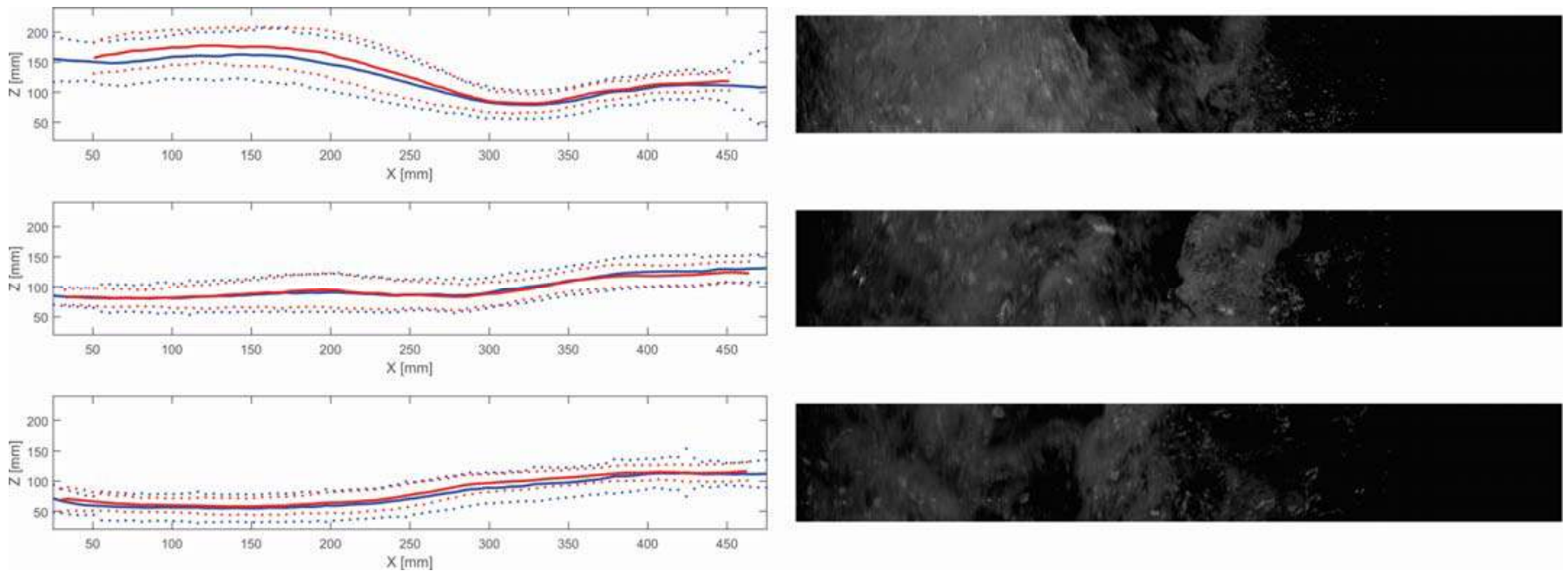

Figure 5. Results of measurements of average turbulent water surface heights (left: solid line), standard deviation of the surface heights (left: dotted line, $\pm 1 \sigma$ ), and corresponding images recorded in sequence by the secondary camera (right). Results are shown for LIDAR (blue) and triangulation method (red). Above: CS1, middle: CS2 and below: CS3.
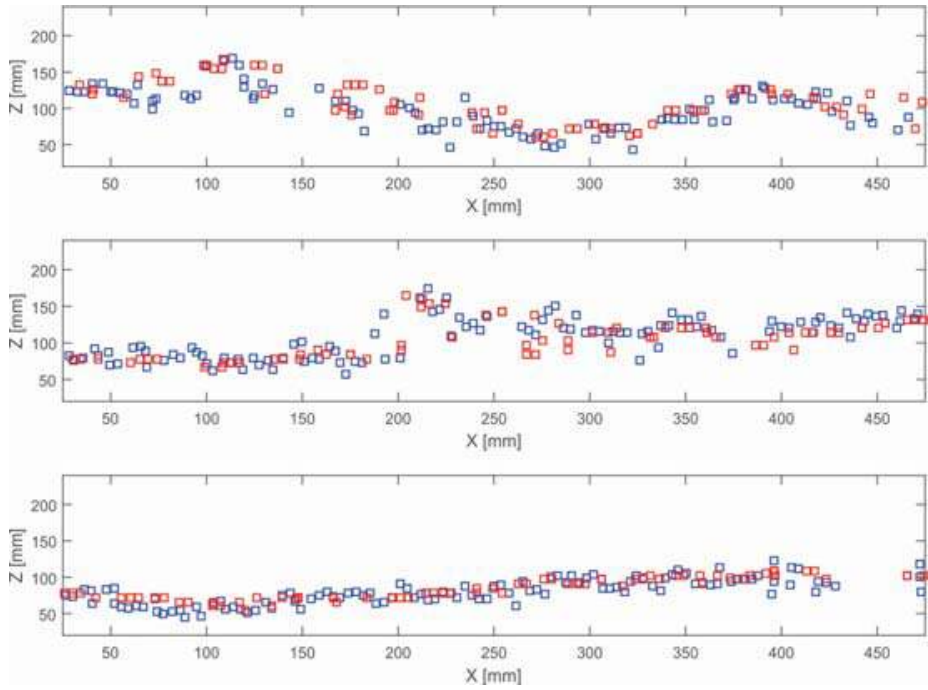

Figure 6. Samples of turbulent water surface heights. Blue: LIDAR and red: triangulation method. Above: CS1, middle: CS2 and below: CS3.

Figure 6 shows examples of turbulent water surface profiles. Both laser ranging methods failed to provide valid measurements for all measurement positions as described in section 3 "Analysis". The amount of rejected measurements for LIDAR was $14.6 \%$ for CS1, $14.9 \%$ for CS2 and $13.2 \%$ for CS3 and $12.2 \%$ for CS1, $7.2 \%$ for CS2 and $9.2 \%$ for CS3 for laser triangulation.

The amount of rejected measurements provide an estimate of how laser light originating from successive reflections at different air/water boundaries such as droplets, bubbles and waves is detected by LIDAR and the laser triangulation high-speed camera. It is clear that these values clearly do not agree despite both methods using the same laser beam i.e., from the LIDAR.

The cross sections differ among each other on the amount of turbulence at the surface. CS1 has a more turbulent surface because it is located nearer to the confluence than CS2 and CS3. CS3 is located the farthest downstream from the confluence and had the least turbulent surface. Surface turbulence did not have a significant affect on the amount of rejected measurements for both methods. LIDAR had a greater number of rejected measurements than the laser triangulation method. However, the number of rejected measurements is heavily influenced by the settings of the high-speed camera's image processing algorithm (it is not possible to alter the LIDAR algorithm).

Commercial LIDAR devices require high thresholds to trigger single distance acquisitions. Laser beam scattering on fog is usually not sufficient to trigger acquisition except in the case of the largest droplets. The mechanism responsible for triggering a commercial LIDAR on a water surface is specular reflection. The laser beam is reflected from the water surface in several consecutive reflections. A reflection capable of triggering the LIDAR must be reflected back to the LIDAR with sufficient intensity. However, the point from where such a reflection originates depends on the previous reflection or series of previous reflections. The laser beam from the LIDAR is sent and received at the same angle of mirror 
rotation. Because of the short time it takes for a laser pulse to travel from the LIDAR be reflected and travel back the turbulent water surface for a single point LIDAR measurement is measured as stationary. Only laser light returning from the point of impact and the narrow spatial angular section of the radius vector from the LIDAR to the impact point is detected by the LIDAR's photodetector (Fig. 1: dark yellow region in the cross section). Reflected beams originating from other positions are rejected.

The reflections of the laser beam to the high-speed camera are recorded differently. For a single image, the high-speed camera lens collects light reflected in the direction of the lens. These reflected beams originate from a larger volume, illuminated by successive specular reflections from the surface of the water. Therefore, the laser triangulation method offers slightly poorer localisation in comparison with LIDAR. The high-speed camera's ability to record weak reflected beams is compromised by the need for high frequency image acquisition and a very short image integration time, which leads to high noise and low image quality.

Direct comparison of single measured points by LIDAR (Red circles) and laser triangulation (green circles) is shown in Figure 7 . Three manually selected typical images recorded by the high-speed camera are shown. Successive specular reflections are visible as a cloud of multiple dark spots, whereas laser triangulation detects the approximate centre of the brightest point in the cloud. Notably, the measurements provided by the LIDAR are not in the same locations as the centres of intensities of the reflections (Fig. 1). The difference is, however, not large and this mismatch is caused by differences in the observation positions. The reflected beams, returning to the LIDAR are reflected from a different position than those reflected to the laser triangulation high-speed camera. This observation confirms the assumption that specular reflections are the most important factor relating to laser ranging measurements.

The current triangulation method algorithm is based on locating the brightest area in the image originating from laser beam scattering (see section 4 "Analysis"). Since image smoothing (Gaussian filter) is performed before the maximum search, the filter width affects the resultsThe wider the filter, the more robust are the results but the spatial resolution is smaller. Therefore, the parameters of the laser triangulation method represent a compromise between resolution and spatial noise reduction.

The average intensities for all three of the measured cross sections recorded the laser triangulation by the high-speed camera are shown in Figure 8. The average of over 700 acquired images corresponding to two LIDAR scans was obtained. The results differ slightly from those presented in Figure 5 due to a shorter integration time. For instance, a local increase in the water level observed for CS3 on the right is a consequence of non-stationary local flow turbulence. The second important information in Figure 8 is the
Laser ranging measurements of turbulent water surface- 6 depiction of an average laser beam scattering through the droplets (middle image), steep waves (left and right sides of middle and below images) and entrapped bubbles below the surface (centre top and middle image). All these features may be regarded as near-diffuse reflections as discussed in Section 1.2. The width of the contour on the image corresponds to the average depth of light penetration. Thus, the wider the contours the more dynamic is the behaviour of the water.
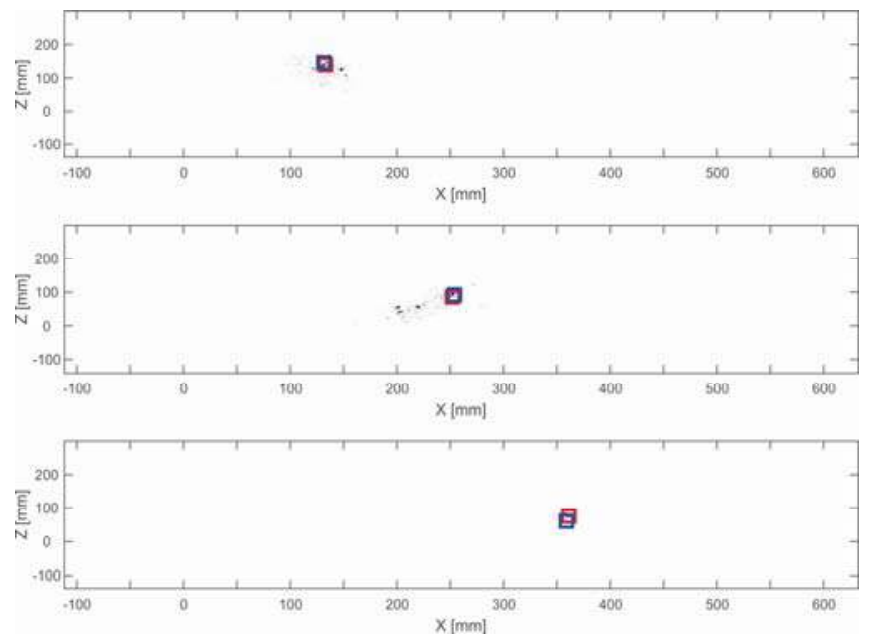

Figure 7. A comparison of LIDAR and image triangulation method results for a single point value in the 2D LIDAR profile and its corresponding high-speed camera image CS2. Red squares show LIDAR measurements. Blue squares show measurement results from the image triangulation method.

Black and grey dots represent the intensity of reflections, detected with the high-speed camera.

In contrast, there is no way to access the algorithm used to process the LIDAR signal since it is a commercial device optimised for measuring solid diffuse surfaces where a single high contrast laser pulse is detected. This means that to understand the LIDAR's performance, it would be necessary to evaluate the time series of the returned LIDAR beam intensity. A comparison with the recorded high-speed camera images would allow such an optimization of the LIDAR performance for the purpose of water surface measurements of turbulent free surface flows.

The experimental setup in this study allows a comparison of each LIDAR measurement (for each scan and angular position) with a corresponding laser triangulation high-speed camera image, recorded at approximately the same time. However, phenomena comparison is limited because laser triangulation high-speed camera images do not show the turbulent water surface from the same location and the secondary camera provides only qualitative data. To understand better the series of specular reflections another high-speed camera should be mounted at the same location. Without the use of a red filter to screen the blue diffuse illumination for the secondary camera, the second high-speed camera would acquire instantaneous turbulent water surface images, illuminated by the blue light. In this new configuration, 
synchronising both cameras and a comparison with the LIDAR results should provide a better understanding of the scattering process and LIDAR operation, how and when the LIDAR detects the turbulent water surface air/water boundary and the roles of flying droplets, foam or bubbles below the surface for turbulent open flow surface measurements.

Statistical methods applied to the whole data set would also provide additional insight when comparing both laser ranging methods.
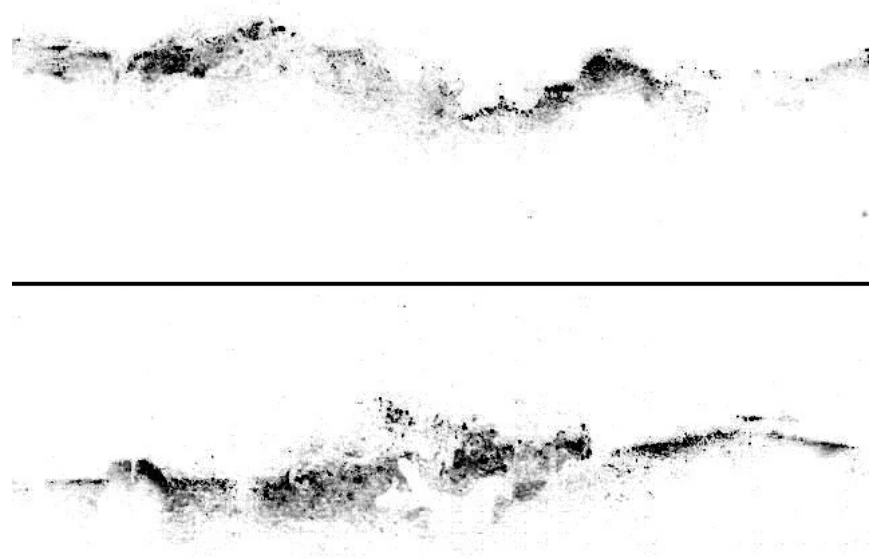

Figure 8. Average intensities of acquired laser triangulation high-speed camera images sequence. Above: CS1, middle: CS2 and below: CS3.

\section{Conclusions}

This paper presents the first known comparison of two laser ranging methods for profiling turbulent open flows: LIDAR and laser triangulation. Both methods were applied to a $90^{\circ}$ confluence flow with high Reynolds and Froude numbers. Analysis was performed such that the LIDAR laser beam was also used for laser triangulation with a high-speed camera. Both measurements methods were operated simultaneously. The study showed that:

- performance of both methods is comparable when making height measurements of turbulent open surfaces,

- both methods estimate equally well the average turbulent open surface height,

- neither method provides the same instantaneous height measurements for every position on the surface profile,
Laser ranging measurements of turbulent water surface- 7 - LIDAR rejects more measurements in the case of less turbulent surfaces than the laser triangulation method in its current configuration which rejected more measurements for highly turbulent surfaces, and

- LIDAR is more localized than the laser triangulation method.

In this study, a slow secondary camera located near the LIDAR was used to provide qualitative data of the flow, however, the use of an additional synchronised high-speed camera would provide a better understanding how flying droplets, foam and bubbles trapped below the surface affect the performance of the LIDAR and laser triangulation methods. Such a configuration would also allow for optimizing of the LIDAR algorithm used for measuring water surface measurements for turbulent free surface flows.

Acknowledgments: The authors acknowledge the financial support from the state budget by the Slovenian Research Agency (Programmes No. P2-0392, P2 0270, P2 - 0167 and P2 - 0401).

\section{REFERENCES}

[1] H. Chanson. Hydraulics of Aerated Flows: Qui Pro Quo?, Journal of Hydraulic Research, 51 (3): 223-243, 2013

[2] M. C. Amann, T. Bosch, M. Lescure, R. Myllylä, M. Rioux. Laser ranging: a critical review of usual techniques for distance measurement, Optical Engineering, 40 (1): 10-19, 2001

[3] J. Shan, C. K. Toth. Topographic Laser Ranging and Scanning: Principles and Processing, CRC Press, 2008

[4] R. S. Wang, Y. Hu, H. Y. Wu, J. Wang. Automatic extraction of building boundaries using aerial LiDAR data. Journal of Applied Remote Sensing, 10 (1): 16-22, 2016

[5] C. E. Blenkingsopp, M. A. Mole, I. L. Turner, W. L. Peirson. Measurements of the time-varying free-surface profile across the swash zone obtained using an industrial LIDAR. Coastal Engineering, 57: 1059-1065, 2010

[6] M. J. Allis, W. L. Peirson, M. L. Banner. Application of LIDAR as a measurement tool for waves. Proceedings of the Twenty-first International Offshore and Polar Engineering Conference. USA, Maui, Hawaii, International Society of Offshore and Polar Engineers: 19-24, 2011

[7] C. E. Blenkingsopp, I. L. Turner, M. J. Allis, W. L. Peirson, L.E. Garden. Application of LiDAR technology for measurement of time-varying free-surface profiles in a laboratory wave flume. Coastal Engineering, 68: 1-5, 2012

[8] P. Westfeld, H. G. Maasa, K. Richter, R. Weiß. Analysis and correction of ocean wave pattern induced systematic coordinate errors in airborne LiDAR bathymetry, ISPRS Journal of Photogrammetry and Remote Sensing, 128: 314-325, 2017

[9] C. Mulsow, M. Schulze, P. Westfeld. An optical triangulation method for height measurements on nonstationary water surfaces, International Archives of the 
Photogrammetry, Remote Sensing and Spatial Information Sciences: 213-217, 2006

[10] C. Mulsow, H. G. Maas, P. Westfeld, M. Schulze. Triangulation methods for height profile measurements on instationary water surfaces, Journal of Applied Geodesy, 2 (1): 21-29, 2008.

[11] M. Y. Brazhnikov, G. V. Kolmakov, A. A. Levchenko and L. P. Mezhov-Deglin. Observation of capillary turbulence on the water surface in a wide range of frequencies, EPL (Europhysics Letters), 5 (4): 510-516, 2002

[12] E. Mignot, N. Riviere, R. Perkins, A. Paquier. Flow patterns in a four-branch junction with supercritical flow. Journal of Hydraulic engineering, 134 (6): 701-713, 2008

[13] M. M. Pinto Coelho. Experimental determination of free surface levels at open-channel junction. Journal of Hydraulic Research 53, 3: 394-399, 2015

[14] J. D. Nobrega, H. E. Schulz, D.Z. Zhu, Free Surface Detection in Hydraulic Jumps through Image Analysis and Ultrasonic Sensor Measurements. 5th IAHR International Symposium on Hydraulic Structures. Brisbane, Australia: 1-8, 2014

[15] M. Schwalt, W. H. Hager. Experiments to supercritical junction flow. Experiments in Fluids, 18: 429-437, 1995

[16] L. S. Nanía, R. Gonzalo, M. Gómez. Influence of Channel Width on Flow Distribution in Four-Branch Junctions with Supercritical Flow: Experimental Approach. Journal of Hydraulic Engineering, 140: 7788, 2014

[17] H. W. Jensen, S. R. Marschner, M. Levoy, P. Hanrahan. A practical model for subsurface light transport. Proceedings of ACM SIGGRAPH 2001: 511518, 2001

[18] G. Rak, M. Hočevar, F. Steinman. Measuring water surface topography using laser scanning. Flow Measurement and Instrumentation, 48 (11), 35-44, 2017

[19] M. Jezeršek, J. Možina. High-speed measurements of foot shape based on multiple-laser-plane triangulation. Optical Engineering, 48(11): 113604-1 - 113604-8, 2009 